\title{
Improving Effective Magnetic Resonance Imaging (MRI) Application in Soft Tissue Wrist Injury
}

\author{
Alfred P. Yoon, MD, Alexandra L. Mathews, BS, Helen E. Huetteman, BS, \\ Brett F. Michelotti, MD, and Kevin C. Chung, MD, MS
}

Introduction: Magnetic resonance imaging (MRI) for soft-tissue wrist injury may be overprescribed, contributing to ineffective health care resource use. We aimed to discern predictive factors that may improve MRI's application in soft-tissue wrist injury.

Methods: We conducted a retrospective chart review of adults who underwent MRIs for possible softtissue wrist injury between June 2009 and June 2014. Clinical data and treatment recommendations before and after MRI were analyzed. If the MRI-directed treatment recommendation was different from before MRI, the MRI was noted to have influenced the patient's treatment (Impact MRI).

Results: Among 140 MRI scans, 39 (28\%) impacted treatment recommendation. Twenty-six Impact MRIs were ordered by hand surgeons, whereas 13 were ordered by referring physicians $(P=.001)$. More Impact MRIs were found when an MRI was ordered for patients younger than 36 years $(P=.01)$, within 6 weeks of symptom onset $(P=.03)$, to question a specific anatomic injury $(P=.0001)$, or by a board-certified hand surgeon $(P=.001)$. Adjusting for other covariates, these 4 clinical factors were identified as independent predictive factors to Impact MRIs.

Conclusions: MRIs for soft-tissue wrist injuries may more likely change management when the patient is younger, ordered within 6 weeks of symptom onset, and prescribed with a specific differential diagnosis. Referral to a hand surgeon should be considered before wrist MRI for the following patients: history of hand surgery/trauma, older than $\mathbf{3 6}$ years likely due to confounding chronic wrist changes, symptomatic for more than 6 weeks, and without clear differential diagnoses for the symptoms. (J Am Board Fam Med 2018;31:795-804.)

Keywords: Differential Diagnosis, Magnetic Resonance Imaging, Soft Tissue Injuries, Surgeons, Wrist Injuries

The United States' per capita expenditure on health care has increased over the past 15 years and is higher than any other country. ${ }^{1}$ Expenditures on

This article was externally peer reviewed.

Submitted 29 October 2017; revised 1 June 2018; accepted 5 June 2018.

From the Section of Plastic Surgery, Department of Surgery, The University of Michigan Medical School, Ann Arbor (APY, HEH); Stritch School of Medicine, Loyola University, Chicago, IL (ALM); Department of Surgery, Division of Plastic Surgery, University of Wisconsin Health System, Madison, WI (BFM); Section of Plastic Surgery, Assistant Dean for Faculty Affairs, The University of Michigan Medical School, Ann Arbor (KCC).

Funding: Research reported in this publication was supported by a PSF Pilot Research Grant awarded to the authors in July, 2016, an award from the National Institute of Arthritis and Musculoskeletal and Skin Diseases Midcareer Investigator Award in Patient-Oriented Research (2K24 AR053120-06) to Dr. Kevin Chung. The content is solely the responsibility of the authors and does not necessarily represent the official views of the National Institutes of Health. medical imaging are approaching $\$ 100$ billion annually, with magnetic resonance imaging (MRI) accounting for the majority of this expense. MRI overuse has not been proven to improve patient outcomes, but can increase health care system burden. Nevertheless, studies show continued overutilization of advanced imaging techniques by physicians. $^{2-4}$

To decrease unnecessary expenditures, the American College of Radiology (ACR) developed the Appropriateness Criteria in 1993. Researchers report a low utilization of the ACR Appropriateness Criteria

Conflict of interest: none declared.

Corresponding author: Kevin C. Chung, MD, MS, Section of Plastic Surgery, University of Michigan Health System, 2130 Taubman Center, SPC 5340, 1500 E. Medical Center Drive, Ann Arbor, MI, 48109-5340 (E-mail: kecchung@ umich.edu). 
by physicians ordering imaging studies, especially MRIs. ${ }^{4-6}$ Unfortunately, the criteria determined by the ACR do not provide detailed guidelines for many clinical scenarios. ${ }^{3}$ As a result, many physicians are unaware of the appropriate indications for ordering an MRI. ${ }^{3}$

Studies have examined the use of MRI in patients with nonspecific hip, knee, and low back pain. ${ }^{2,4,5,7-10}$ Lehnert et $\mathrm{al}^{4}$ specifically highlighted the need for tools to help primary care physicians improve advanced diagnostic imaging decision making. Wrist pain is an analogous musculoskeletal complaint; however, few have explored the appropriate use of MRI in patients with wrist pain. ${ }^{10-15}$ Diagnosing the cause of wrist pain is challenging because of the complex bony and ligamentous anatomy, and the broad spectrum of potential wrist pathology. ${ }^{13}$ In addition, research suggests that compared with those of specialists, musculoskeletal medical training is inadequate in nonspecialist training programs. ${ }^{14-17}$ Hartzell et al ${ }^{18}$ evaluated the management of patients with common hand disorders including nerve compression, benign hand masses, Dupuytren's disease, and trigger finger or thumb before referral to a hand specialist. The authors found that, before hand surgeon referral, a correct diagnosis was made only $34 \%$ of the time. In addition, of the patients who underwent advanced diagnostic imaging, $90 \%$ of the imaging studies were unnecessary. This study suggests that the role of advanced diagnostic imaging in common hand and wrist conditions needs further scrutiny to prevent ineffective use of health care resources.

The aim of this study was to discern predictive factors that may improve MRI's application in soft tissue wrist injury and identify patient cohorts that may benefit from a hand specialist referral before an MRI is ordered. Furthermore, we compared the effects on clinical decision making in patients with suspected soft-tissue wrist injury from wrist MRIs ordered by both referring physicians and hand surgeons. We hypothesize that most wrist MRIs ordered to investigate soft-tissue wrist injury do not influence treatment recommendation, and that certain demographic and clinical factors may increase meaningful use of wrist MRIs. The results from this study aim to provide physicians with clinical decision support to direct appropriate use of MRI in patients presenting with wrist pain.

\section{Methods}

After obtaining institutional review board approval, we conducted a retrospective review of medical records of all patients between ages 18 and 65 years who visited the University of Michigan Health System between June 2009 and June 2014 for injury to 1 of the following wrist ligaments: scapholunate ligament (SL), triangular fibrocartilage complex (TFCC), or lunotriquetral ligament. We also included patients who were diagnosed with distal radioulnar joint (DRUJ) instability, ulnocarpal abutment, or wrist pain presumably from ligamentous injury.

Patients with wrist injuries were identified using the University of Michigan Medical School research tool, DataDirect. This tool was used to identify specific International Classification of Disease, version 9 (ICD-9) diagnosis codes within a patient's medical record (Appendix). Patients were included if they underwent at least 1 wrist MRI investigating a potential wrist ligament injury and had at least 1 documented clinical examination and/or standard radiographic assessment of the wrist before the MRI. Patients who had symptoms or signs of additional pathology (eg, concern for fracture, infection, inflammatory or crystalline arthropathy, neoplasm, compression neuropathy, or severe degenerative arthritis) or complex prior hand surgical history were excluded from the study. We designed these exclusion criteria to specifically identify patients with only soft-tissue or ligamentous wrist injuries without confounding influence from other hand pathologies. Excluded patients were identified either through physical examination or radiographic imaging. Relevant demographic factors including age, sex, body mass index, smoking status, prior hand surgery/trauma, duration of conservative pain management, health insurance, and existing comorbid conditions were recorded. We used the Charlson Comorbidity Index for each patient to quantify comorbidity.

All MRI reports, radiograph reports, and electronic medical records of patients who met inclusion criteria over the study period were compiled. Only MRI scans with a $1.5 \mathrm{~T}$ magnet were analyzed to eliminate bias. We did not distinguish between noncontrast MRI and gadolinium contrast MRI because previous studies demonstrate that contrast does not improve diagnostic accuracy. ${ }^{19}$ From the MRI report, we gathered information regarding 
Figure 1. Flowchart of Cohort Selection. MRI, magnetic resonance imaging.

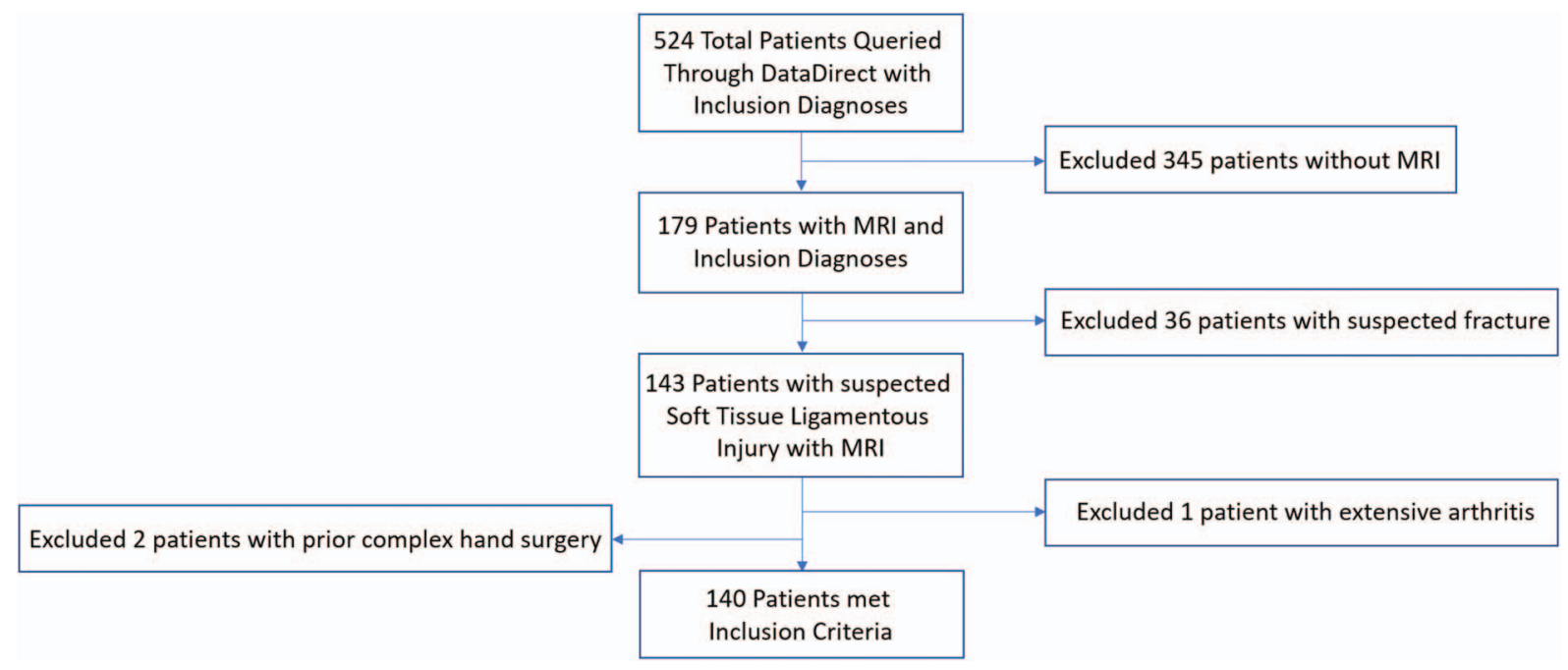

study indications, clinician ordering the MRI, reported MRI diagnosis, and order date. Because most clinicians consider 6 weeks the demarcation between acute and chronic soft-tissue ligament injuries, ${ }^{20}$ we used the 6 -week time frame as a point of interest in our analyses. We classified hand fellowship trained plastic and orthopedic surgeons into the "hand surgeon group," whereas other physicians (eg, family medicine, internal medicine, physical medicine and rehabilitation, rheumatology, etc.) were classified into the "referring physician group."

We reviewed relevant clinical history, context of the diagnosis, and proposed patient treatment plan before and after the MRI study. Patients were grouped by MRI study indication (to question a specific injury vs general wrist pain) and specific study indication groups including question SL ligament injury, question TFCC injury, question DRUJ instability, or question other ligamentous injury. Each MRI was analyzed individually. If the MRI-directed treatment recommendation was different from the treatment recommendation before the wrist MRI, we assumed that the MRI influenced the patient's treatment and defined this as an "Impact MRI." To make this designation, we compared pre-MRI treatment recommendations from patients' medical records to post-MRI treatment recommendations. If no clear treatment recommendation was recorded in the chart before the MRI, 1 hand surgeon from our team, blinded to the results of the MRI, retrospectively formulated the treatment recommendation (operative, nonoperative, or equivocal). This recommendation was compared with the reported treatment recommendation after the wrist MRI. In both scenarios, an MRI scan was considered unnecessary if 1) the treatment recommendation before the wrist MRI was identical to the treatment recommendation after the wrist MRI, or 2) the MRI was normal.

Patient characteristics were analyzed using the Student $t$ tests for continuous variables and Fisher exact test for categorical variables. Patient characteristics and MRI study indications were compared between Impact MRI versus Nonimpact MRI groups and hand surgeon versus referring physician groups. The changes in treatment recommendations (operative, nonoperative, or equivocal) before and after MRI were analyzed in detail. Multivariate logistic regression modeling was applied to isolate clinical factors associated with Impact MRIs. Statistical analysis was performed using the software $\mathrm{R}$ (University of Auckland, Auckland, New Zealand) with statistical significance set at 0.05 .

\section{Results}

A total of 524 patients were initially queried; after excluding patients without MRIs, and those with suspected fractures, complex hand surgery, or arthritis, 140 patients met our inclusion criteria (Figure 1). Eighty-seven (62.1\%) were female participants and $53(37.9 \%)$ were male (Table 1). About half $(n=86)$ of patients were seen in specialty care 


\begin{tabular}{|c|c|c|c|c|c|c|c|}
\hline \multirow[b]{2}{*}{ Number of patients } & \multicolumn{2}{|c|}{ All Patients } & \multicolumn{2}{|c|}{ Impact MRI Group } & \multicolumn{2}{|c|}{$\begin{array}{c}\text { Non-Impact MRI } \\
\text { Group }\end{array}$} & \multirow[t]{2}{*}{$P$-Value } \\
\hline & 140 & & 39 & $28 \%$ & 101 & $72 \%$ & \\
\hline Age & 37 & & 33 & $(30-37)^{*}$ & 39 & $(36-41)^{*}$ & $.01^{\dagger \neq}$ \\
\hline \multicolumn{8}{|l|}{ Gender } \\
\hline Male & 53 & $38 \%$ & 14 & & 39 & & $.85^{\S}$ \\
\hline Female & 87 & $62 \%$ & 25 & & 62 & & \\
\hline BMI & 28.2 & & 28.2 & & 27.8 & & $.86^{\dagger}$ \\
\hline Obesity & 48 & $34 \%$ & 13 & $33 \%$ & 35 & $35 \%$ & $1.00^{\S}$ \\
\hline Smokers & 21 & $15 \%$ & 7 & $18 \%$ & 14 & $14 \%$ & $.60^{\S}$ \\
\hline \multicolumn{8}{|l|}{ Referring provider } \\
\hline UMHS & 122 & $87 \%$ & 33 & $85 \%$ & 99 & $88 \%$ & $.56^{\S}$ \\
\hline Non-UMHS & 18 & $13 \%$ & 6 & $15 \%$ & 12 & $12 \%$ & \\
\hline Previous hand surgery/trauma & 41 & $29 \%$ & 10 & $26 \%$ & 31 & $31 \%$ & $.68^{\S}$ \\
\hline Charlson Comorbidity Index & 0.31 & & 0.1 & & 0.3 & & $.15^{\S}$ \\
\hline Prior therapy/splinting/injections & 87 & $62 \%$ & 21 & $54 \%$ & 66 & $65 \%$ & $.25^{\S}$ \\
\hline Insured in healthcare & 133 & $95 \%$ & 37 & $95 \%$ & 96 & $95 \%$ & $1.00^{\S}$ \\
\hline MRI timing ( $<6$ weeks of symptoms) & 48 & $34 \%$ & 19 & $49 \%$ & 29 & $29 \%$ & $.03^{* \S}$ \\
\hline
\end{tabular}

BMI, body mass index; MRI, magnetic resonance imaging; UMHS, University of Michigan Health System.

$* 95 \%$ confidence interval (CI).

${ }^{\dagger}$ Student $t$-test.

${ }^{\ddagger}$ Statistical significance.

${ }^{\S}$ Fisher exact test.

offices, whereas the remaining were treated in primary care offices. Of those who were seen in specialty care offices, most (57\%) presented initially to the emergency department or to a primary care physician and were subsequently referred to a specialty care physician for consultation before their wrist MRI.

Of the 140 MRI scans included, only 39 scans (28\%) impacted patient treatment recommendation. Of the 101 Nonimpact MRI studies, 14 were normal MRIs. The Impact MRI group was younger than the Nonimpact MRI Group (mean age, 33 years [95\% CI, 29.5-36.5] vs 38.5 years [ $95 \% \mathrm{CI}$, 36.4-40.6], respectively; $P=.01$ ). Compared to Nonimpact MRIs, Impact MRIs were more often ordered within 6 weeks of symptom onset (48.7\% vs $28.7 \% ; P=.03)$. Impact MRIs were also ordered more often to question a specific injury rather than generic wrist pain $(71.8 \%$ in Impact MRI vs $35.6 \%$ in Nonimpact MRI; $P=.0012$; Table 2) and ordered by hand surgeons compared to referring physicians ( $38 \%$ vs $18 \% ; P=.0048)$. Remaining demographic factors were not different between the 2 cohorts.

Comparing the demographic variables between patients in the hand surgeon and referring physician cohorts, none were statistically different aside from previous hand surgery or trauma (Table 3). The hand surgeon cohort had significantly more previous hand surgery or trauma than the referring physician cohort $(40.9 \%$ vs $18.9 \% ; P=.005)$. Similar to the comparison between study indications in the Impact MRI versus Nonimpact MRI groups, MRIs ordered by hand surgeons were more often performed with a specific diagnosis in mind compared with those ordered by referring physicians (65.2\% vs $35.1 \% ; P=.0006$; Table 4 ). More Impact MRIs ordered by referring physicians shifted treatment recommendations from nonoperative to operative (46.2\% vs $15.4 \% ; P=.056)$. Significantly more Nonimpact MRIs ordered by referring physicians did not change the pre-MRI recommendation of nonoperative management as compared with those ordered by hand surgeons $(90.2 \%$ vs 50.0\%; $P<.001$; Table 5).

Multivariate logistic regression was used to confirm whether the clinical factors identified from the bivariate analyses were independent predictive factors for an Impact MRI when adjusted for other variables (Table 6). Ordering the MRI within 6 weeks of symptom onset (OR, 2.59; $P=.026)$ had the highest predictive value for an Impact MRI. On the contrary, ordering a wrist MRI without a specific diagnosis was the strongest negative predictor 
Table 2. Comparison of Impact vs Nonimpact MRI

\begin{tabular}{|c|c|c|c|c|c|}
\hline \multirow{2}{*}{$\begin{array}{l}\text { Variable } \\
\text { Total patients }\end{array}$} & \multicolumn{2}{|c|}{ Impact MRI } & \multicolumn{2}{|c|}{ Nonimpact MRI } & \multirow[t]{2}{*}{$P$-Values } \\
\hline & 39 & $28 \%$ & 101 & $72 \%$ & \\
\hline \multicolumn{6}{|l|}{ Ordering physician } \\
\hline Hand surgeon & 26 & $67 \%$ & 40 & $40 \%$ & $.0048^{\star \dagger}$ \\
\hline Referring physician & 13 & $33 \%$ & 61 & $60 \%$ & \\
\hline \multicolumn{6}{|l|}{ Study indication } \\
\hline Wrist pain & 11 & $28 \%$ & 60 & $64 \%$ & $.0012^{* \dagger}$ \\
\hline Question of specific injury & 28 & $72 \%$ & 41 & $36 \%$ & \\
\hline DRUJ stability & 2 & & 1 & & \\
\hline Scapholunate injury & 17 & & 7 & & \\
\hline TFCC injury & 3 & & 12 & & \\
\hline Other ligamentous injury & 6 & & 21 & & \\
\hline
\end{tabular}

DRUJ, distal radioulnar joint; MRI, magnetic resonance imaging; TFCC, triangular fibrocartilage complex.

${ }^{*}$ Fisher exact test.

${ }^{\dagger}$ Statistical significance.

of an Impact MRI (OR, 0.39; $P=.036)$ followed by an MRI ordered by a referring physician (OR, 0.43 ; $P=.052)$ and older patient age (OR, 0.97; $P=$ $.067)$.

\section{Discussion}

Soft-tissue wrist injuries are challenging to diagnose, which, as evidenced by the prevalence of Nonimpact MRIs in our study, may explain physi- cian tendency to overprescribe wrist MRIs in patients with suspected soft-tissue wrist injury. Although MRIs are highly sensitive in detecting anatomic derangement, clinical examination with or without radiograph alone may provide a diagnosis at a lower cost. We demonstrated that $72 \%$ of wrist MRIs ordered by physicians, including hand surgeons and other physician types, over the course of our study period did not impact patient treat-

Table 3. Baseline Demographics Comparison between Hand Surgeon and Referring Physician Patient Cohorts

\begin{tabular}{|c|c|c|c|c|c|}
\hline \multirow[b]{2}{*}{ Number of patients } & \multicolumn{2}{|c|}{ Hand Surgeon Group } & \multicolumn{2}{|c|}{$\begin{array}{c}\text { Referring Physician } \\
\text { Group }\end{array}$} & \multirow[t]{2}{*}{$P$-Value } \\
\hline & 66 & & 74 & & \\
\hline Age & 36 & & 38 & & $.15^{*}$ \\
\hline \multicolumn{6}{|l|}{ Gender } \\
\hline Male & 22 & $33 \%$ & 31 & $42 \%$ & $.39^{\dagger}$ \\
\hline Female & 44 & $67 \%$ & 43 & $58 \%$ & \\
\hline BMI & 28.8 & & 27.9 & & $.47^{*}$ \\
\hline Obesity & 24 & $36 \%$ & 24 & $32 \%$ & $.60^{\dagger}$ \\
\hline Smokers & 9 & $14 \%$ & 12 & $16 \%$ & $.81^{\dagger}$ \\
\hline \multicolumn{6}{|l|}{ Referring provider } \\
\hline UMHS & 112 & $85 \%$ & 66 & $89 \%$ & $.61^{\dagger}$ \\
\hline Non-UMHS & 10 & $15 \%$ & 8 & $11 \%$ & \\
\hline Previous hand surgery/trauma & 27 & $41 \%$ & 14 & $19 \%$ & $.005^{\dagger \neq}$ \\
\hline Charlson Comorbidity Index & 0.24 & & 0.36 & & $.24^{*}$ \\
\hline Prior therapy/splinting/injections & 44 & $67 \%$ & 43 & $58 \%$ & $.38^{\dagger}$ \\
\hline Insured in healthcare & 62 & $94 \%$ & 71 & $96 \%$ & $.71^{\dagger}$ \\
\hline MRI timing ( $<6$ weeks of symptoms) & 27 & $41 \%$ & 21 & $28 \%$ & $.15^{\dagger}$ \\
\hline
\end{tabular}

BMI, body mass index; MRI, magnetic resonance imaging; UMHS, University of Michigan Health System.

*Student $t$-test.

${ }^{\dagger}$ Fisher exact test.

${ }^{\ddagger}$ Statistical significance. 
Table 4. Comparison of MRI Indications Ordered by Hand Surgeons and Referring Physicians

\begin{tabular}{lcccc}
\hline Variables & Hand Surgeon MRI & \multicolumn{2}{c}{$\begin{array}{c}\text { Referring Physician } \\
\text { MRI }\end{array}$} & $P$-Values \\
\hline Total patients & 66 & $47 \%$ & 74 & $53 \%$ \\
Study indication & 23 & $35 \%$ & 48 & $65 \%$ \\
Wrist pain & 43 & $65 \%$ & 26 & $35 \%$ \\
Question of specific injury & 3 & & 0 & $.0006^{* \dagger}$ \\
DRUJ stability & 20 & & 4 & \\
Scapholunate injury & 7 & & 8 & \\
TFCC injury & 13 & & 14 & \\
Other ligamentous injury & & & & \\
\hline
\end{tabular}

DRUJ, distal radioulnar joint; MRI, magnetic resonance imaging; TFCC, triangular fibrocartilage complex.

*Statistical significance.

${ }^{\dagger}$ Fisher exact test.

ment recommendation. Wrist MRIs that do not change clinical management are an ineffective use of health care resources that increase costs to payers. Our findings suggest that larger multicenter cohort studies observing wrist MRI use are warranted to develop guidelines that increase meaningful use of wrist MRIs.

Analyzing baseline demographic data, our sample included a significantly greater number of female than male patients ( 87 vs 53 , respectively; $P<$ $.01)$. The true incidence of ligamentous injury in the hand is unknown. However, a cadaveric analysis of 96 wrists by Lee et $\mathrm{al}^{21}$ discovered that at least $35 \%$ of the wrists had some degree of scapholunate injury. We are not aware of any proven increased rate of wrist soft-tissue injuries in women compared with men, and the higher prevalence of women in our study likely is an incidental finding. Examining the demographic variables between the Impact MRI versus Nonimpact MRI groups and the hand surgeon versus referring physician groups, there was no statistical difference in gender distribution between respective cohorts; therefore, this incidental finding likely does not affect the study's conclusions.

Our analysis suggests several predictive clinical factors may increase the likelihood of an Impact MRI. Based on bivariate analysis, being younger than 36 years was associated with increased odds of an MRI that changed clinical management. Adjusting for other covariates, age did not reach statistical significance in the multivariate logistic regression but trended toward being an independent predictor of Impact MRIs (Table 6; $P=.067$ ). With age,

Table 5. Pre-MRI to Post-MRI Recommendation Changes in Impact and Non-Impact MRI Studies

\begin{tabular}{|c|c|c|c|c|c|c|}
\hline \multirow{2}{*}{$\begin{array}{l}\text { Pre/Post MRI Recommendations } \\
\text { Impact MRI }\end{array}$} & \multirow{2}{*}{$\begin{array}{c}\text { All } \\
\text { Patients } \\
39\end{array}$} & \multicolumn{2}{|c|}{$\begin{array}{l}\text { Hand Surgeon } \\
\text { Cohort Patients }\end{array}$} & \multicolumn{2}{|c|}{$\begin{array}{c}\text { Referring } \\
\text { Physician Cohort } \\
\text { Patients }\end{array}$} & \multirow[t]{2}{*}{$P$-Values } \\
\hline & & 26 & & 13 & & \\
\hline Non-op $\rightarrow$ Op & 10 & 4 & $15 \%$ & 6 & $46 \%$ & $.056^{*}$ \\
\hline Op $\rightarrow$ Non-op & 0 & 0 & $0 \%$ & 0 & $0 \%$ & 1.00 \\
\hline $\mathrm{Eq} \rightarrow$ Non-op & 19 & 15 & $58 \%$ & 4 & $31 \%$ & .18 \\
\hline $\mathrm{Eq} \rightarrow \mathrm{Op}$ & 10 & 7 & $27 \%$ & 3 & $23 \%$ & 1.00 \\
\hline $\mathrm{Op} \rightarrow \mathrm{Eq}$ & 0 & 0 & $0 \%$ & 0 & $0 \%$ & 1.00 \\
\hline Non-op $\rightarrow$ Eq & 0 & 0 & $0 \%$ & 0 & $0 \%$ & 1.00 \\
\hline Nonimpact MRI & 101 & 40 & & 61 & & \\
\hline Non-op $\rightarrow$ Non-op & 75 & 20 & $50 \%$ & 55 & $90 \%$ & $<.001^{*}$ \\
\hline $\mathrm{Op} \rightarrow \mathrm{Op}$ & 21 & 16 & $40 \%$ & 5 & $8 \%$ & .20 \\
\hline $\mathrm{Eq} \rightarrow \mathrm{Eq}$ & 5 & 4 & $10 \%$ & 1 & $2 \%$ & .08 \\
\hline
\end{tabular}

Eq, equivocal; MRI, magnetic resonance imaging; Non-op, non-operative; Op, operative. *Statistical significance. 
Table 6. Multivariate Regression of Predictive Factors to Impact MRI

\begin{tabular}{lccc}
\hline & Adjusted OR & $95 \%$ CI & $P$-Values \\
\hline Age & 0.97 & $(0.93-1.00)$ & .067 \\
MRI Timing (<6 weeks of symptoms) & 2.59 & $(1.12-5.99)$ & $.026^{*}$ \\
Study indication: No specific diagnosis & 0.39 & $(0.16-0.94)$ & $.036^{*}$ \\
Referring physician & 0.43 & $(0.18-1.00)$ & $.052^{*}$ \\
\hline
\end{tabular}

CI, confidential interval; MRI, magnetic resonance imaging; OR, odd ratio.

*Statistical significance.

increased degenerative changes occur in the wrist that may further obscure diagnosis of soft-tissue wrist injury. ${ }^{22}$ MRI's high sensitivity to any softtissue or bony injuries detect chronic degenerative changes in the wrist, and it may be difficult to distinguish between chronic and acute problems. Given the relative absence of chronic wrist changes in younger patients, a younger age may lend more specific findings in the wrist MRI that directly pertain to the acute wrist pain, which increases both diagnostic and clinical decision-making value.

Another predictive variable that may increase clinical value of an MRI is the time of study from initial symptom onset. Most clinicians consider 6 weeks as the demarcation between acute and chronic soft-tissue ligament injuries. ${ }^{20}$ Moreover, it has been reported that early direct repair of ligaments in the acute stage results in better outcomes. ${ }^{20}$ Similar to the effect of aging, as an acute injury becomes chronic, the wrist undergoes more irreversible changes that limit surgical options. For instance, acute SL injuries, depending on the grade of injury, have various surgical options such as direct repair, closed-reduction percutaneous pinning, bone-ligament-bone reconstruction, dynamic tendon transfers, among others. However, if a SL injury becomes chronic and progresses to scapholunate lunate advanced collapse, only salvage operations remain. Ordering an MRI within 6 weeks of symptom onset may be challenging because of variations in timing of patient presentation and the period needed for conservative management attempts before advanced imaging studies. However, special tests of the wrist such as the Watson's test, lunotriquetral ballotment test, or piano key sign, in conjunction with wrist rdiographs may help the physician determine the need for a wrist MRI in a timelier fashion.

In addition, we found that MRIs investigating specific anatomic injuries, as opposed to generic wrist pain, resulted in more studies that changed clinical management. Although there is a lack of previous research on this concept, we postulate that this is an indirect result of improved patient selection for MRIs accomplished through formulating a differential diagnosis. To devise such possible diagnoses, the provider must conduct a thorough history, physical examination, and review of radiographs, which will naturally improve patient selection for wrist MRIs. After this process, the MRI ideally becomes a confirmatory staging tool to guide treatment depending on the severity or other associated injuries of the already suspected diagnosis.

Finally, we found that the type of ordering physician, hand surgeon or referring physician, is an independent predictor of Impact MRIs even after adjusting for potentially confounding covariates. The only significantly different demographic factor between the hand surgeon and referring physician cohorts was previous hand surgery or trauma. This finding is expected because patients with previous hand surgeries or traumas are likely already followed by hand surgeons, and may be more likely to present to hand surgeons should new problems arise. However, prior hand injuries or surgeries would decrease the specificity of MRIs due to baseline aberrant anatomy, which arguably should lead to fewer Impact MRIs rather than the increased number of Impact MRIs found in our study.

Further analysis between the 2 cohorts revealed that hand surgeons more frequently ordered wrist MRIs with a specific diagnosis in question compared to referring physicians, which was identified as a positive predictor of Impact MRIs in our analysis between Impact MRIs and Nonimpact MRIs. Adjusting for age, MRI timing, and study indication, the type of physician ordering the MRI still resulted as an independent predictor of Impact MRIs. Two potential explanations for these findings are 1) better patient selection for MRIs by hand surgeons, and 2) hesitation to offer surgery as 
a recommendation by referring physicians compared to hand surgeons. These hypotheses are supported by the proportionately greater number of pre-MRI nonoperative patients in the referring physician group who were subsequently recommended surgery after undergoing MRI, presumably by a hand surgeon (Table 5). In addition, significantly more pre-MRI nonoperative patients in the referring physician group remained nonoperative after the MRI when compared to its counterpart (Table 5). Patients in this subset could have been managed conservatively without obtaining an MRI, representing a cohort of patients that was selected for an MRI erroneously. The hand surgeon cohort also had a nontrivial number of patients $(n=20)$ who were managed nonoperatively both before and after the MRI, although still less than those in the referring physician cohort $(n=55)$.

The limitations of this study include its retrospective nature and its homogenous patient sample in a community adjacent to a large tertiary referral academic center. These factors may limit the generalizability of the study conclusions to other practice settings and geographical regions. Another limitation of this study is that the MRI protocol could not be standardized because patients frequently underwent MRIs at outside imaging centers; therefore, MRI studies with gadolinium contrast or MRI arthrograms were not differentiated in this study. Additional studies involving various MRI protocols are needed. If the patients received imaging studies from an outside institution, the images were scanned into the electronic medical records for interpretation by University of Michigan radiologists. We were unable to control for possible interpretation differences among various University of Michigan radiologists, but assume that they had sufficient experience to accurately interpret a wrist MRI. Radiologists were not blinded from clinical context given ethical and practical concerns of possible increase in false negative or positive reads. Lastly, because this study included patients with soft-tissue wrist injury, our findings cannot be generalized to patients with other conditions such as rheumatologic, infectious, neoplastic, or bony etiologies. Nonetheless, this study is instrumental to providing insight on the use of wrist MRI for soft-tissue ligament injuries among all physician types.

\section{Conclusions}

Soft-tissue wrist injury is challenging to diagnose, and, resultantly, providers tend to overprescribe MRIs. With careful patient selection based on history, physical examination, and radiographs, more impactful MRIs that affect treatment can be ordered. MRIs for patients younger than 36 years, MRIs ordered within 6 weeks of symptom onset, and MRIs ordered with a specific differential diagnosis may increase the probability of a wrist MRI that will change patient management. Conversely, a referral to a hand specialist before obtaining an MRI should be considered for older patients, patients symptomatic for longer than 6 weeks, patients with a prior history of hand surgery or trauma, and cases of uncertain anatomic etiology of the wrist pain. Further large multicenter cohort studies are warranted to establish clear clinical guidelines for effective and appropriate use of MRIs in soft tissue wrist injury. In a value-driven medical system, a costly and unnecessary MRI study may be avoided with better patient selection and earlier referrals to a hand specialist.

To see this article online, please go to: http://jabfm.org/content/ 31/5/795.full.

\section{References}

1. Issa K, Jauregui JJ, McElroy M, et al. Unnecessary magnetic resonance imaging of hips: An economic burden to patients and the healthcare system. J Arthroplasty 2014;29:1911-4.

2. Bautista AB, Burgos A, Nickel BJ, et al. Do clinicians use the American College of Radiology Appropriateness criteria in the management of their patients? AJR Am J Roentgenol 2009;192:1581-5.

3. Hendee WR, Becker GJ, Borgstede JP, et al. Addressing overutilization in medical imaging. Radiology 2010;257:240-5.

4. Lehnert BE, Bree RL. Analysis of appropriateness of outpatient CT and MRI referred from primary care clinics at an academic medical center: How critical is the need for improved decision support? J Am Coll Radiol 2010;7:192-7.

5. Boden SD, McCowin PR, Davis DO, et al. Abnormal magnetic-resonance scans of the cervical spine in asymptomatic subjects. A prospective investigation. J Bone Joint Surg Am 1990;72:1178-84.

6. Flynn TW, Smith B, Chou R. Appropriate use of diagnostic imaging in low back pain: A reminder that unnecessary imaging may do as much harm as good. J Orthop Sports Phys Ther 2011;41:838-46.

7. Carragee E, Alamin T, Cheng I, et al. Are first-time episodes of serious LBP associated with new MRI findings? Spine J 2006;6:624-35. 
8. Chou R, Fu R, Carrino JA, Deyo RA. Imaging strategies for low-back pain: Systematic review and metaanalysis. Lancet 2009;373:463-72.

9. Keeney JA, Nunley RM, Adelani M, et al. Magnetic resonance imaging of the hip: Poor cost utility for treatment of adult patients with hip pain. Clin Orthop Relat Res2014;472:787-92.

10. Roberts TT, Singer N, Hushmendy S, et al. MRI for the evaluation of knee pain: Comparison of ordering practices of primary care physicians and orthopaedic surgeons. J Bone Joint Surg Am 2015; 97:709-14.

11. Andersson JK, Andernord D, Karlsson J, et al. Efficacy of magnetic resonance imaging and clinical tests in diagnostics of wrist ligament injuries: A systematic review. Arthroscopy 2015;31:2014-20.e2.

12. Couzens G, Daunt N, Crawford R, et al. Positive magnetic resonance imaging findings in the asymptomatic wrist. ANZ J Surg 2014;84(7-8):528-532.

13. Ruston J, Konan S, Rubinraut E, et al. Diagnostic accuracy of clinical examination and magnetic resonance imaging for common articular wrist pathology. Acta Orthop Belg 2013;79:375-80.

14. Freedman KB, Bernstein J. The adequacy of medical school education in musculoskeletal medicine. J Bone Joint Surg Am 1998;80:1421-7.
15. Freedman KB, Bernstein J. Educational deficiencies in musculoskeletal medicine. J Bone Joint Surg Am 2002;84:604-8.

16. Matzkin E, Smith EL, Freccero D, et al. Adequacy of education in musculoskeletal medicine. J Bone Joint Surg Am 2005;87:310-4.

17. Scher DL, Boyer MI, Hammert WC, et al. Evaluation of knowledge of common hand surgery problems in internal medicine and emergency medicine residents. Orthopedics 2011;34(7):e279-e281.

18. Hartzell TL, Shahbazian JH, Pandey A, et al. Does the gatekeeper model work in hand surgery? Plast Reconstr Surg 2013;132(3):381e-386e.

19. Schädel-Höpfner M, Iwinska-Zelder J, Braus T, et al. MRI versus arthroscopy in the diagnosis of scapholunate ligament injury. J Hand Surg Br 2001; 26:17-21.

20. Rohman EM, Agel J, Putnam MD, et al. Scapholunate interosseous ligament injuries: A retrospective review of treatment and outcomes in 82 wrists. J Hand Surg Am 2014;39:2020-6.

21. Lee DH, Dickson KF, Bradley EL. The incidence of wrist interosseous ligament and triangular fibrocartilage articular disc disruptions: A cadaveric study. J Hand Surg Am 2004;29:676-84.

22. Mikić ZD. Age changes in the triangular fibrocartilage of the wrist joint. J Anat 1978;126(Pt 2):367384. 


\section{Appendix}

\begin{tabular}{ll}
\hline ICD-9 Code & \multicolumn{1}{c}{ Description } \\
\hline 719.43 to 719.44 & Wrist pain \\
728.4 & Laxity of ligament \\
833.01 & DRUJ Instability (closed) \\
833.11 & DRUJ Instability (open) \\
842.00 & Sprain of wrist, unspecified site \\
842.01 & Sprains and strains wrist and hand; \\
& wrist; carpal (joint) \\
842.02 & $\begin{array}{c}\text { Sprain of radiocarpal (ligament) of } \\
\text { wrist }\end{array}$ \\
842.09 & $\begin{array}{c}\text { Sprains and strains of wrist and hand; } \\
\text { distal radioulnar joint }\end{array}$ \\
905.7 & Late effect of sprain and strain without \\
& mention of tendon injury \\
959.3 & Other and unspecified injury to elbow \\
& forearm and wrist \\
\hline
\end{tabular}

ICD-9, International Classification of Disease, version 9. 